\title{
Response of the DHCAL to Hadrons and Positrons
}

\section{Burak Bilki ${ }^{1}$}

On behalf of the CALICE Collaboration

Argonne National Laboratory

Argonne, IL, USA

University Of Iowa

Iowa City, IA, USA

E-mail: burak-bilki@uiowa.edu

\begin{abstract}
We have constructed a prototype Digital Hadron Calorimeter (DHCAL) using Resistive Plate Chambers (RPCs) as the active elements. The RPCs are read out digitally (1-bit) by $1 \times 1 \mathrm{~cm}^{2}$ pads. The DHCAL consists of 38 alternating layers of steel and RPCs followed by a 14 layer tail catcher (TCMT), also read out using RPCs. With each layer consisting of 9,216 channels, the entire detector contains 350,208 (DHCAL) $+129,024(\mathrm{TMCT})=479,232$ readout channels (world record in calorimetry). Each active layer contains three $32 \times 96 \mathrm{~cm}^{2}$ RPCs.

In this paper we report on results from a first look at the DHCAL data to assess the calorimetric properties of the DHCAL. We present preliminary results from the analysis of both positron and pion events of momenta between 2 and $60 \mathrm{GeV} / \mathrm{c}$. These results are considered to be a first validation of the viability of the DHCAL concept.
\end{abstract}

XI workshop on Resistive Plate Chambers and Related Detectors (RPC2012)

INFN-Laboratori Nazionali di Frascait, Italy

February 5-10, 2012

1 Speaker 


\section{Introduction}

For our purposes, a 2-glass plate design was developed and optimized for calorimetry. The outside of each glass is coated with a resistive paint in order to apply high voltage (HV). A front-end-board containing the readout pads is pressed against the anode side of the chamber. The details about the construction and calibration of the DHCAL are provided in [1-2].

We collected more than 35 million events in five test beam campaigns at the Fermilab Test Beam Facility between October 2010 and December 2012. The DHCAL was extensively tested with $1-60 \mathrm{GeV} / \mathrm{c}$ secondary beam (a momentum selected mixture of muons, positrons and pions) and with the $120 \mathrm{GeV} / \mathrm{c}$ primary proton beam.

\section{Analysis Strategy}

The data read out from the DHCAL contain the hit position information, the time stamp of the individual hits and the time stamp from the trigger and timing unit. The hits in each layer are combined into clusters using a nearest-neighbor approach. If two hits share a common edge, they are assigned to the same cluster. The event selection requires not more than 1 cluster in Layer 1 with at most four hits. This selection assures that upstream interactions are not included. The requirements of at least three active layers and no hits in the two outermost pads in any layer select clean events related to beam particles and events that have transverse containment, respectively.

The beam is a momentum selected mixture of muons, pions and positrons. This analysis is based on a topological particle identification utilizing the high segmentation of the DHCAL. The particle identification algorithms are described in detail in [3].

Once the particles are identified, the distributions of total number of hits in the DHCAL are obtained for pions and positrons separately. Figure 1 shows the distributions for $20 \mathrm{GeV}$ pions (left) and positrons (right). The entire spectrum is fit to a Gaussian with no corrections or calibrations applied. These fits are used as input to the calorimetric measurements.
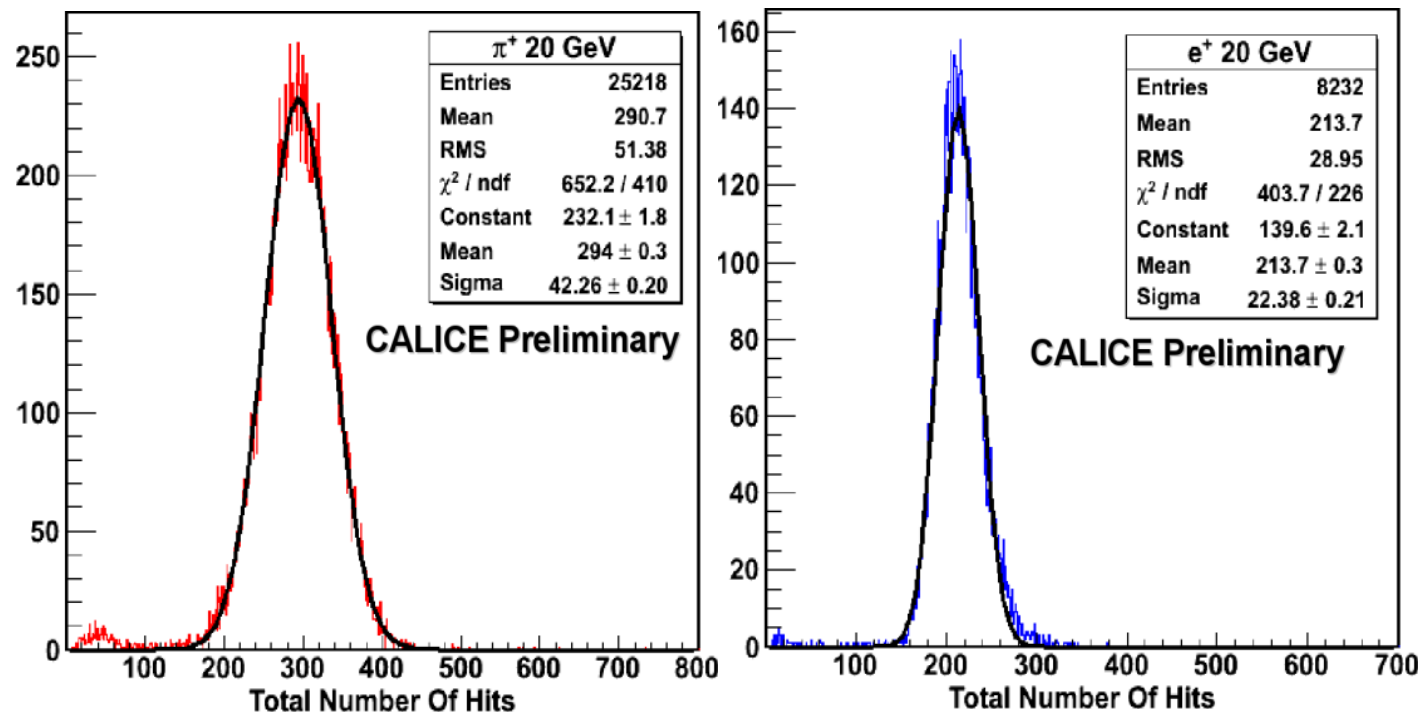

Figure 1. Distribution of total number of hits for $20 \mathrm{GeV}$ pions (left) and positrons (right). 


\section{DHCAL Response to Hadrons}

The mean response of the DHCAL to pions is shown in Fig. 2a. The response is linear up to $25 \mathrm{GeV}$, and at $32 \mathrm{GeV}$ the response is somewhat lower than expected. This decrease of the response was later attributed to changes in the calibration, due to fluctuations in the ambient temperature. Therefore, $32 \mathrm{GeV}$ data point is not included in the linear fit $(\mathrm{N}=\mathrm{aE}$ where $\mathrm{N}$ is the total number of hits and $\mathrm{E}$ is the beam energy). Except for the $32 \mathrm{GeV}$ point, the deviation between the point predicted by the line and the data point is within $\pm 2 \%$. These results have been obtained without calibration of the response of the DHCAL. Figure $2 b$ shows the hadronic energy resolution of the DHCAL with the current particle identification algorithms. The $32 \mathrm{GeV}$ points are again excluded from the fits. The fits represent the data well and for the longitudinally contained pions -that have no hits in the last two layers- a stochastic term of approximately $55 \%$ and a constant term of $7.5 \%$ is achieved. The measurements are within $1-2$ $\%$ of predictions [4] based on the simulation of the large-size DHCAL prototype using the vertical slice test (VST) results [4-8].

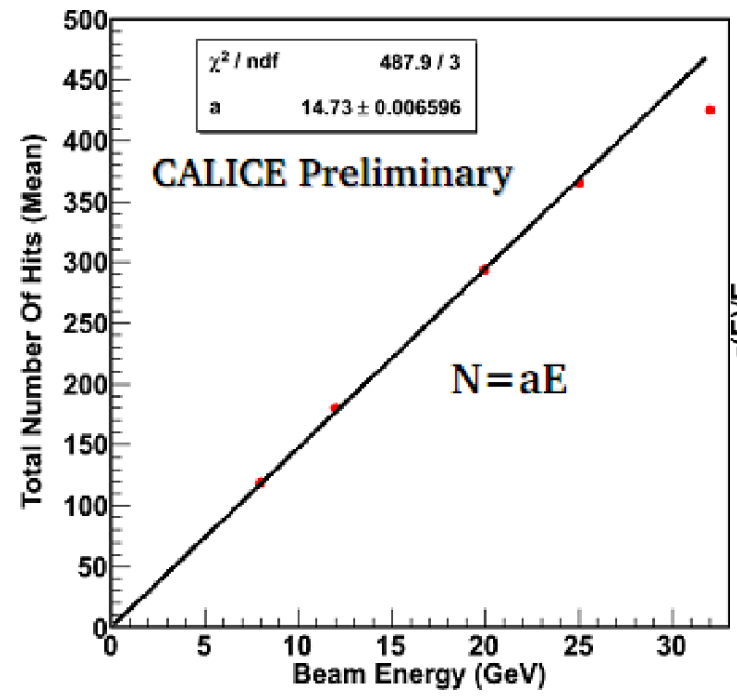

(a)

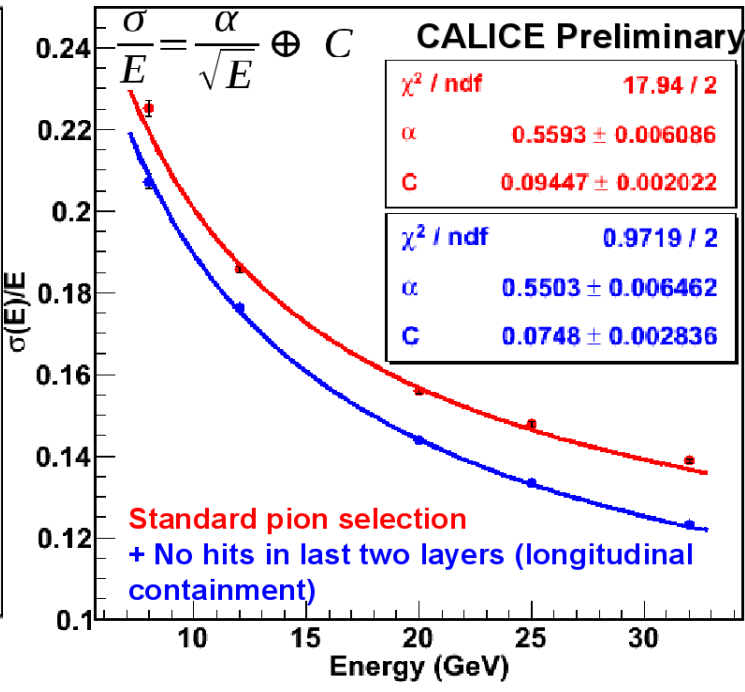

(b)

Figure 2. The response of the DHCAL to pions (a) and the hadronic energy resolution (b) for all pions (red) and the longitudinally contained pions (blue).

\section{DHCAL Response to Positrons}

The mean response of the DHCAL to identified positrons is shown in Fig. 3a. The response is fit with the nonlinear function $\mathrm{N}=\mathrm{a}+\mathrm{bE} \mathrm{E}^{\mathrm{m}}$. The fit describes the data well and is in accordance with the predictions in the VST results of positron showers [7]. In order to measure the electromagnetic energy resolution of the DHCAL with the positron response corrected for non-linearity, the total number of hits for each positron event is mapped into its corresponding energy value using the fit function in Fig. 3a. Then these reconstructed energy spectra are used to calculate the energy resolutions. Figure $3 b$ shows the electromagnetic energy resolution for both uncorrected and corrected values. 

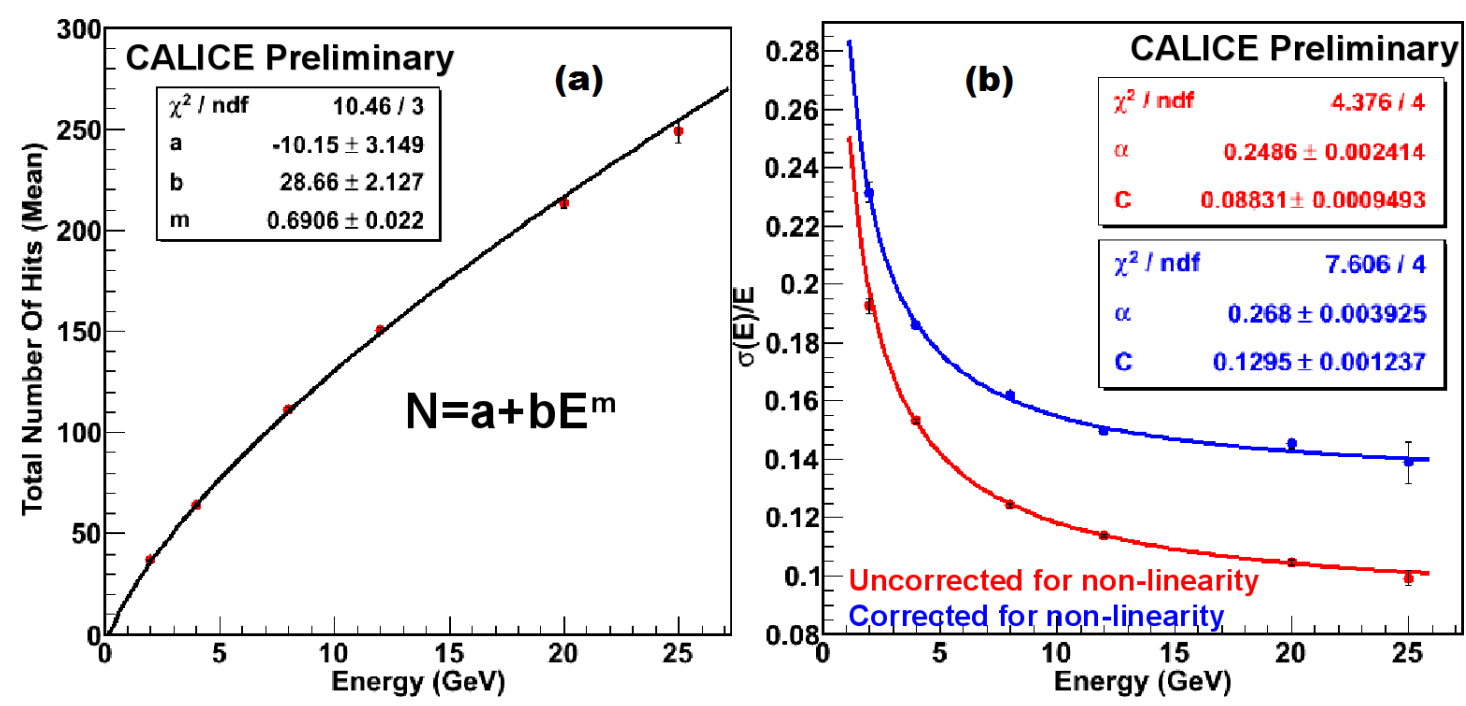

Figure 3. Mean response of DHCAL to positrons (a) and the electromagnetic energy resolutions (b) for the uncorrected (red) and corrected (blue) response.

\section{Conclusions}

We have completed five successful test beam campaigns where Run-3 included the CALICE Silicon-Tungsten Electromagnetic Calorimeter (ECAL) placed in front and Run-5 was performed with minimal absorber material in between the RPC layers. The particle identification algorithms defined within this text provide sufficiently well discrimination at momenta above $6 \mathrm{GeV} / \mathrm{c}$. However, the complications in the event topologies at low energies require further studies to integrate these energies into the calorimetric measurements. These new algorithms are expected to improve the current measurements as well. With the present algorithms, a hadronic energy resolution of $\sigma / E=55 \% / \sqrt{E} \oplus 7.5 \%$ and an electromagnetic energy resolution between $24 \%$ and $14 \%$ in the energy range of $2-25 \mathrm{GeV}$ are obtained. These results were obtained without calibration of the individual DHCAL layers.

Further methods are being developed to obtain unbiased samples of pure beam particles and to obtain the DHCAL response not only as an energy measuring calorimeter, but also as a unique source of information of detailed hardonic interactions with unprecedented spatial resolution.

\section{References}

[1] L. Xia, Construction of a Digital Hadron Calorimeter with Resistive Plate Chambers, these proceedings.

[2] J. Repond, Analysis of DHCAL Events, these proceedings.

[3] B. Bilki, http://ilcagenda.linearcollider.org/getFile.py/access?contribId=148\&sessionId=18\&

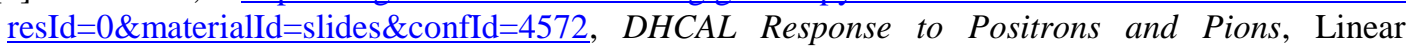
Collider Workshop of the Americas - ALCPG11, 2011.

[4] B. Bilki et.al., Hadron showers in a digital hadron calorimeter, JINST 4 P10008, 2009.

[5] Q. Zhang et.al., Environmental dependence of the performance of resistive plate chambers, JINST 5 P02007, 2010.

[6] B. Bilki et.al., Measurement of the rate capability of Resistive Plate Chambers, JINST 4 P06003, 2009.

[7] B. Bilki et.al., Measurement of positron showers with a digital hadron calorimeter, JINST 4 P04006, 2009.

[8] B. Bilki et.al., Calibration of a digital hadron calorimeter with muons, JINST 3 P05001, 2008. 